\title{
Recubrimientos sol-gel obtenidos por deposición electroforética (EPD) sobre metales
}

\author{
Y. CASTRO, B. FERRARI, R. MORENO, A. DURÁN \\ Instituto de Cerámica y Vidrio, C.S.I.C. Ctra. Valencia Km. 24,300; 28500 Arganda del Rey, Madrid
}

\begin{abstract}
El proceso sol-gel es un método de gran interés para la producción de películas vítreas sobre metales, que actúan como barrera protectora frente a la oxidación y aumentan su resistencia a la corrosión. Se han obtenido recubrimientos inorgánicos e híbridos de distintas composiciones sobre distintos metales usando técnicas como inmersión, centrifugado y pulverización. Aunque estos métodos proporcionan recubrimientos con buenas propiedades los espesores obtenidos son bajos (inferiores a $2 \mathrm{~mm}$ ), lo que limita sus posibilidades de aplicación. La deposición electroforética (EPD) se perfila como una técnica de gran versatilidad para la obtención de recubrimientos homogéneos de mayor espesor. El objetivo de este trabajo es la preparación de recubrimientos sobre metal por EPD a partir de soluciones híbridas de $\mathrm{SiO}_{2}$ (TEOS, MTES) y de suspensiones coloidales de $\mathrm{SiO}_{2}$ en las que las propias soluciones híbridas actúan como medio de dispersión. Tanto las soluciones como las suspensiones se han estudiado mediante medidas de viscosidad, densidad y conductividad. Asimismo, se han estudiado los parámetros físicos asociados a la EPD, tales como el tiempo de deposición y la densidad de corriente, teniendo en cuenta el efecto adicional de formación de capa por inmersión durante la extracción. Los recubrimientos se han caracterizado mediante perfilometría y microscopía óptica, comparando las capas depositadas por inmersión y las obtenidas por EPD.
\end{abstract}

Palabras Clave: Sol-gel, recubrimientos, inmersión, deposición electroforética, sílice coloidal.

Sol-gel coatings obtained from electrophoretic depositions (EPD) on metals

Sol-gel process is a suitable method for the production of glass-like coatings onto metals, acting as barrier against oxidation and providing an increased resistance to corrosion. Hybrid and inorganic coatings of different compositions have been used for coating several metals by using techniques like dipping, spin-coating and spraying. Although these methods lead to good properties the final coatings are restricted by the thin thickness (up to $2 \mu \mathrm{m}$ ), thus limiting the application range. Electrophoretic deposition (EPD) is considered a highly versatile technique for producing homogeneous thicker coatings. The aim of this work deals with the preparation of coatings onto metals by EPD from hybrid silica sols (TEOS, MTES) and from colloidal silica suspensions where the hybrid sols constitute the suspending medium. Both the solutions and the suspensions have been studied in terms of viscosity, density and conductivity. Furthermore, the physical parameters associated to EPD, such as deposition time and current density, have been also studied, taking into account the additional contribution to the layer formed by EPD occurring by dipping during withdrawal. The coatings obtained by dipping and by EPD have been characterised by perfilometry and optical microscopy.

Key words: Sol-gel, coatings, dipping, electrophoretic deposition, colloidal silica.

\section{INTRODUCCIÓN}

La exposición de metales a condiciones de $\mathrm{pH}$ agresivas, a la presencia de gases corrosivos $\left(\mathrm{Cl}_{2}, \mathrm{NH}_{3}\right.$, etc.) o a altas temperaturas, provoca la oxidación o corrosión de los sustratos, así como la degradación de las propiedades de estos materiales, con la consiguiente pérdida de eficiencia en las aplicaciones a las que van destinados. Mediante la utilización de recubrimientos protectores, se intenta aumentar la resistencia al desgaste o abrasión de los sustratos metálicos y su resistencia a la corrosión.

Muchos de los recubrimientos protectores usados en la actualidad son de naturaleza orgánica (poliésteres, resinas epoxy...) [1]. Estos recubrimientos presentan baja durabilidad en condiciones atmosféricas, debido a la permeabilidad del $\mathrm{O}_{2}$ y $\mathrm{H}_{2} \mathrm{O}$ y no se pueden utilizar a temperaturas altas, lo que hace que tengan que volver a ser recubiertos con el paso del tiempo. Esto supone gastos económicos adicionales, problema que se suma a la contaminación producida por el desprendimiento de compuestos orgánicos volátiles durante el proceso de recubrimiento y curado.

Con el fin de conseguir recubrimientos de mayor durabilidad, con alta resistencia al desgaste e incluso con una coloración determinada, es preciso recurrir a sistemas inorgánicos. Las capas producidas por PVD o plasma presentan un buen comportamiento frente a la corrosión, pero el elevado coste de estos procesos de obtención ha impedido su introducción en el mercado.

Mediante la utilización del proceso sol-gel, se pueden conseguir capas densas, sin defectos y resistentes al rayado [2,3]. Este método no es muy costoso, es fácil de aplicar y posee la gran ventaja de permitir la sinterización a temperaturas tan bajas como $500^{\circ} \mathrm{C}$ [4]. Se ha comprobado experimentalmente que estos recubrimientos confieren un carácter protector a los sustratos metálicos [5]. La producción de capas a partir de soluciones híbridas de sílice TEOS/MTES usando la técnica de 
inmersión conduce a espesores críticos de $2 \mu \mathrm{m}$. Se ha comprobado que estos recubrimientos presentan un factor de protección frente a la oxidación y a la corrosión dos órdenes de magnitud más alto que otros tipos de recubrimientos [3, 6, 7].

Sin embargo, el empleo de la técnica de inmersión presenta dos grandes limitaciones: por una parte el espesor crítico se sitúa alrededor de $2 \mu \mathrm{m}$ y, por otra parte, sólo permite recubrir homogéneamente sustratos con geometría axial. Mediante la incorporación de partículas a la solución híbrida, es posible aumentar el espesor del recubrimiento [8]. Por otro lado, la aplicación de la técnica de deposición electroforética (EPD) a este tipo de soluciones/supensiones debería producir recubrimientos de mayor espesor. La EPD presenta importantes ventajas frente a otro tipo de técnicas de deposición de recubrimientos con es la sencillez y versatilidad del método y su bajo costo.

La técnica de deposición electroforética se basa en el fenómeno electrocinético de electroforesis, por el que las partículas en suspensión en un medio líquido migran hacia el electrodo de signo contrario al de su carga superficial, por medio de la aplicación de un campo eléctrico [9, 10, 11]. El aumento del contenido en sólidos y de iones de signo contrarios en el entorno del electrodo provoca la formación de una capa de partículas con cohesión interna, que forma el recubrimiento. La preparación de suspensiones estables es fundamental para favorecer el movimiento de las partículas y obtener capas homogéneas, compactas y sin defectos que, tras la sinterización, permitan mejorar las prestaciones mecánicas del sustrato.

La deposición electroforética puede llevarse a cabo en condiciones potenciostáticas o galvanostáticas. En el primer caso la formación de la capa puede generar un efecto resistivo en el sistema, que provoca una caída de la densidad de corriente con el tiempo, lo que frena la velocidad de deposición pudiendo incluso llegar a detener el proceso. En consecuencia, la masa depositada deja de ser una función lineal del tiempo [9, 12]. En los ensayos a intensidad constante puede también producirse un efecto resistivo en el sistema, que se traduce en un aumento del potencial, dado que la densidad de corriente no varía.

Por otro lado, si los valores de densidad de corriente son elevados se puede producir la descomposición electrolítica del medio y del sustrato, afectando al recubrimiento y a la estabilidad de la suspensión [13].

Todos los estudios sobre recubrimientos por EPD vía sol-gel han sido llevados a cabo utilizando suspensiones de nanopartículas coloidales de $\mathrm{SiO}_{2}, \mathrm{ZrO}_{2}$ y $\mathrm{Al}_{2} \mathrm{O}_{3}$ suspendidas en un medio acuso, por lo que el recubrimiento obtenido está formado por partículas empaquetadas, y para obtener una completa densificación del recubrimiento se requiere aplicar un ciclo de sinterización convencional, con temperatura superiores a $1000^{\circ} \mathrm{C}[14,15]$

Los objetivos de este trabajo son combinar las ventajas del proceso sol-gel, en especial la baja temperatura de sinterización de las capas, con las del proceso de deposición electroforética para conseguir recubrimientos de $\mathrm{SiO}_{2}$ sobre metales más gruesos, sin defectos y con buenas propiedades mecánicas que confieran al sustrato una alta resistencia a la corrosión.

\section{EXPERIMENTAL}

Los soles híbridos de $\mathrm{SiO}_{2}$ se prepararon a partir de tetraetoxisilano (TEOS) y metiltrietoxisilano (MTES), etanol absoluto y agua destilada. Se han usado diferentes ácidos $\left(\mathrm{HCl}, \mathrm{HNO}_{3^{\prime}}\right.$ $\left.\mathrm{H}_{2} \mathrm{SO}_{4}, \mathrm{AcH}\right)$, con los que se ha ajustado el $\mathrm{pH}$ a valores de 2-3.
La formación del sol tiene lugar mediante la reacción de hidrólisis del TEOS y MTES a pH ácido, actuando éste como catalizador de dicha reacción. La reacción de hidrólisis se ha llevado a cabo durante un tiempo de 3 horas a $40^{\circ} \mathrm{C}$. Las relaciones molares utilizadas fueron MTES/TEOS $=40 / 60$ y $\mathrm{H}_{2} \mathrm{O} /$ $($ MTES+ TEOS) $=1,75$ y la concentración del sol fue $200 \mathrm{~g} / 1$. La relación se ha elegido por ser la que conduce a mayores espesores críticos [16]. Partiendo de $\mathrm{pH} 3$ se ha estudiado la influencia del $\mathrm{pH}$ en las propiedades del sol y en las características de los recubrimientos mediante la adición de hidróxido de tetrametil amonio (TMAH) hasta valores de $\mathrm{pH}$ de 5 y 7.

La suspensión con nanopartículas de sílice se preparó adicionando al sol híbrido catalizado con $\mathrm{HNO}_{3}$ a $\mathrm{pH}=3$, una suspensión acuosa comercial de sílice coloidal (Levasil 200S, Bayer, Alemania) con una concentración de partículas de $20 \%$ y cuyo tamaño medio de partícula es de $20 \mathrm{~nm}$, mediante agitación vigorosa. Los contenidos en sólidos preparados fueron 1, 3, 5 y $11,3 \%$ en peso. La tabla 1 muestra las propiedades de estas suspensiones.

TABla 1. CARACTERÍSTICAS DE LAS SUSPENSIONES DE NANOPARTíCULAS DE $\mathrm{SIO}_{2}$ EN EL SOL HÍBRIDO.

\begin{tabular}{|c|ccc|}
\hline $\begin{array}{c}\text { Concentración } \\
\text { de particulas }\end{array}$ & $p H$ & $\begin{array}{c}\text { Viscosidad } \\
(m P a . s)\end{array}$ & $\begin{array}{c}\text { Conductividad } \\
(\mu \mathrm{S} / \mathrm{cm})\end{array}$ \\
\hline $1 \%$ & 3 & 2,2 & 200 \\
$3 \%$ & 3 & 2,5 & 320 \\
$5 \%$ & 3 & 3,0 & 550 \\
$11,3 \%$ & 3 & 4,2 & 2800 \\
\hline
\end{tabular}

La caracterización de las soluciones se realizó determinando la viscosidad con un reómetro de esfuerzo controlado (Haake Rheostress RS50, Alemania) y la conductividad con un conductímetro (LF320 WTW, Alemania).

Como sustratos se utilizaron portamuestras de vidrio y chapas de acero inoxidable AISI 304 cuyas dimensiones son 7 $x 2,5 \mathrm{~cm}^{2}$. Los portamuestras de vidrio se desengrasaron en baño de ultrasonidos con alcohol durante 15 minutos. Las placas de acero inoxidable se desengrasaron con una solución alcalina (P3Emalan5668:P3Emalan0469, Miele, Alemania) durante 5 minutos en ultrasonidos, después en agua destilada durante un minuto y, por último, en agua caliente a $60^{\circ} \mathrm{C}$ durante un minuto.

Los sustratos de vidrio y acero inoxidable se recubrieron utilizando el sol híbrido y el sol híbrido con nanopartículas mediante la técnica de inmersión, utilizando un intervalo de velocidades comprendido entre 9 y $65 \mathrm{~cm} / \mathrm{min}$.

Se ha llevado a cabo un ensayo de voltametría sobre el sol híbrido preparado a $\mathrm{pH}=3$, con el fin de determinar la densidad de corriente límite que puede aplicarse en los ensayos de deposición electroforética sin producir la corrosión de los mismos. Los ensayos se realizaron utilizando como electrodo de trabajo acero inoxidable AISI 304, como contraelectrodo platino $\mathrm{y}$, como electrodo de referencia, un electrodo de calomelanos saturado aplicando una velocidad de barrido de $12 \mathrm{mV} / \mathrm{m}$.

Los ensayos de deposición electroforética sé realizaron con el sol híbrido con y sin partículas. La densidad de corriente se ha mantenido constante mediante una fuente de potencia Labconco (mod. 433-3250, USA). Se han utilizado placas de acero inoxidable AISI 304 como electrodo de trabajo y como contraelectrodo, manteniendo una superficie de conducción de $6,25 \mathrm{~cm}^{2}$ y una separación entre electrodos de $2 \mathrm{~cm}$.. Las densidades de corriente aplicadas están comprendidas entre 0,1 y $3,8 \mathrm{~mA} / \mathrm{cm}^{2}$. 
Los recubrimientos se secaron a temperatura ambiente y se sinterizaron a $450^{\circ} \mathrm{C}$ durante 15 minutos en flujo de $\mathrm{N}_{2}$ y aire.

Los recubrimientos se caracterizaron por microscopía óptica y el espesor de las capas se midió por perfilometría (Talystep, UK) en el caso de los recubrimientos obtenidos sobre portamuestras de vidrio, y por gravimetría y perfilometría, para los obtenidos sobre acero inoxidable AISI 304.

Dado que se trata de ensayos con soluciones sol-gel, la extracción de los sustratos tras la deposición por EPD conlleva la formación de una capa adicional que depende de la velocidad de extracción y de los parámetros de la solución [17]. Para tener en cuenta ambas contribuciones al espesor, se diseño un equipo, construido en el laboratorio del ICV, que consta de una fuente de potencia, un sistema de agitación, - en este caso una placa agitadora -, para mantener la homogeneidad de la suspensión, y un sistema que permite extraer el electrodo de trabajo y el contraelectrodo a una velocidad constante (figura1). De esta forma, el espesor debido a la electroforesis se obtiene restando al espesor total medido tras la EPD, el espesor debido a la inmersión. El espesor total obtenido es la suma de dos contribuciones, una correspondiente a la inmersión y otra a la propia EPD:

$$
\mathrm{e}_{\text {total }}=\mathrm{e}_{\text {inmersión }}+\mathrm{e}_{\mathrm{EPD}}
$$

El grado de densificación de los recubrimientos sobre acero inoxidable AISI 304 preparados utilizando los soles preparado a $\mathrm{pH}=3,5$ y 7 se evaluó mediante la técnica de inmersión a través de curvas de polarización. La fuente potenciostática utilizada para llevar a cabo las voltametría fue Gamry (mod. CMS105, USA) aplicando una velocidad de barrido de $12 \mathrm{mV} / \mathrm{m}$ en $\mathrm{NaCl} 0.6 \mathrm{~N}$ a temperatura ambiente.

\section{RESULTADOS Y DISCUSIÓN}

La preparación de los soles híbridos se llevó a cabo con diferentes tipos de catalizadores ácidos, $-\mathrm{HCl}, \mathrm{HNO}_{3}, \mathrm{H}_{2} \mathrm{SO}_{4} \mathrm{y}$ ácido acético-, con el fin de estudiar cómo afectaba cada uno de ellos a los soles y al aspecto de los recubrimientos. En todos los casos se prepararon recubrimientos sobre portamuestras de vidrio y con distintas velocidades de inmersión utilizando el sol de concentración $200 \mathrm{~g} / 1$. Con todos los catalizadores se consiguieron soles homogéneos y recubrimientos compactos y uniformes tras el tratamiento térmico, pero sólo con los soles catalizados en $\mathrm{HCl}$ y $\mathrm{HNO}_{3}$ se consiguieron recubrimientos totalmente transparentes.

La estabilidad de los soles preparados con $\mathrm{HCl}$ y $\mathrm{HNO}_{3}$ se evaluó midiendo la variación de la viscosidad en función del tiempo. En la figura 2 se puede observar que ambos soles mantienen valores de viscosidad menores de $4 \mathrm{mPa}$. s durante tiempos prolongados, aunque en el caso de sol con $\mathrm{HNO}_{3}$ se consiguen tiempos de 30 días frente a los 12 del sol catalizado con $\mathrm{HCl}$.

La figura 3 representa el espesor de las capas, homogéneas y sin defectos, en función de la velocidad de inmersión, alcanzándose en ambos casos un espesor crítico de $2 \mu \mathrm{m}$.

Por tanto, ambos ácidos pueden utilizarse como catalizadores para obtener soles estables, homogéneos y transparentes, y recubrimientos uniformes. Sin embargo, es bien conocido el efecto negativo que tienen los iones cloruro sobre los sustratos metálicos. La presencia de estos iones provoca la ruptura de la película pasivante, aumentando la densidad de corriente y acelerando el proceso de corrosión [18]. Dado que el uso de $\mathrm{HNO}_{3}$ no supone incorporación de cloruros y prolonga la esta-

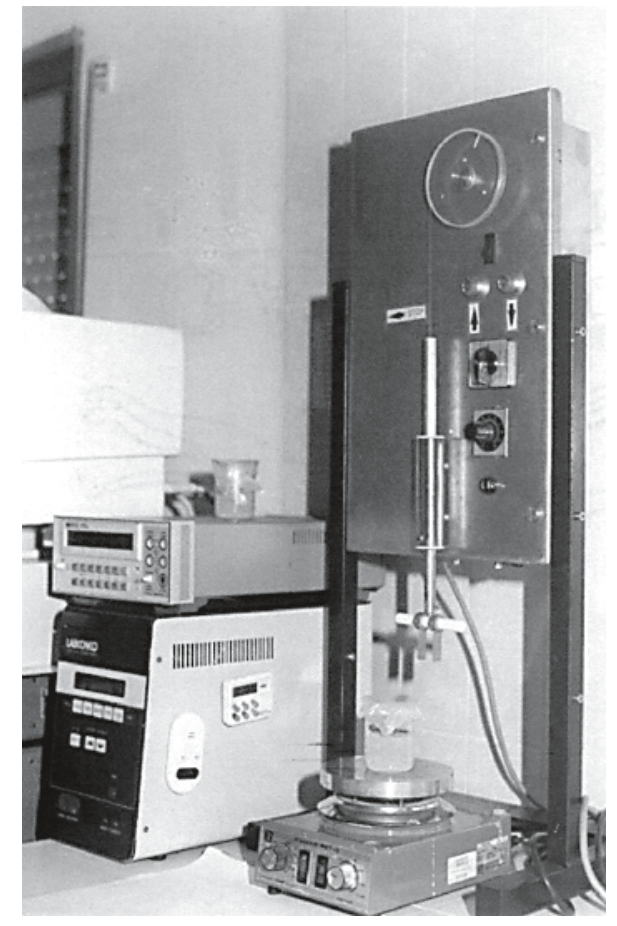

Figura 1. Equipo diseñado para los ensayos de deposición electroforética con velocidad de extracción controlada.

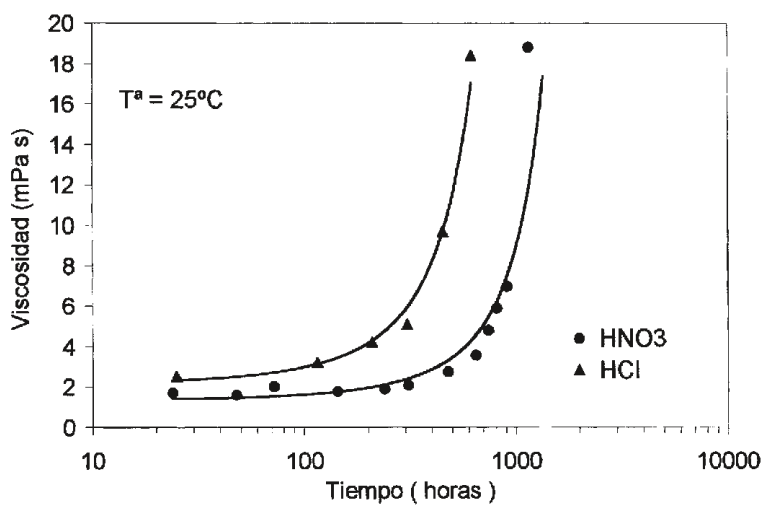

Figura 2 Variación de la viscosidad con el tiempo de soles híbridos de TEOS $/$ MTES $=40 / 60$ catalizados con $\mathrm{HCl}$ y $\mathrm{HNO}_{3}$

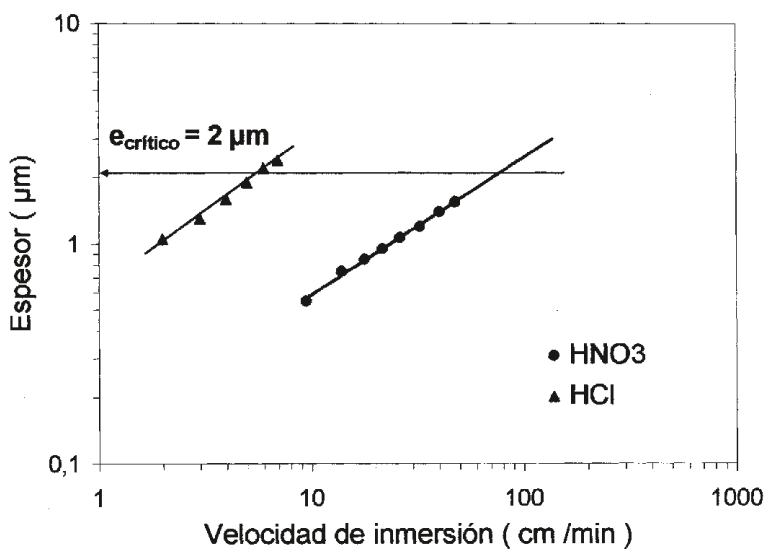

Figura 3 Variación del espesor con la velocidad de extracción de recubrimientos obtenidos a partir de soles híbridos de TEOS/MTES $=40 / 60$ catalizados con $\mathrm{HCl}$ y $\mathrm{HNO}_{3}$ 
bilidad del sol, se ha seleccionado este ácido como catalizador para los restantes experimentos.

Se prepararon recubrimientos sobre acero inoxidable AISI 304, utilizando la técnica de inmersión a partir del sol híbrido catalizado con $\mathrm{HNO}_{3}(\mathrm{pH}=3)$ y de los soles a los que se modificó el $\mathrm{pH}$ añadiendo TMAH hasta valores de 5 y 7 . En la figura 4 se muestran las curvas de polarización en $\mathrm{NaCl} 0,6$ $\mathrm{N}$ de los sustratos recubiertos con los soles de distinto $\mathrm{pH}$, junto con la del acero inoxidable AISI 304 sin recubrir, todos ellos tratados a $450^{\circ} \mathrm{C}$ durante 15 minutos. Las curvas de polarización muestran que los recubrimientos obtenidos a partir de los soles con pH 5 y 7 están activos en todo el intervalo de potencial, mientras que el recubrimiento obtenido para el sol con $\mathrm{pH}=3$ se encuentra pasivo, con una densidad de corriente de pasivación de aproximadamente $10^{-8} \mathrm{~mA} /$ $\mathrm{cm}_{2}$ y un potencial de picadura de $300 \mathrm{mV} / \mathrm{SCE}$. Estos ensayos demuestran que el incremento del $\mathrm{pH}$ reduce el efecto protector de los recubrimientos, debido posiblemente al incremento de defectos en la red o a la baja densidad de las capas, obteniéndose el mejor comportamiento con el sol a pH $=3$.

Por tanto, los soles híbridos catalizados con $\mathrm{HNO}_{3}$ a $\mathrm{pH}=$ 3 fueron seleccionados para los primeros ensayos de deposición electroforética, que se realizaron aplicando densidades de corriente entre 0,1 a $3,8 \mathrm{~mA} \mathrm{~cm}_{-2}$ durante un tiempo de deposición de una hora, utilizando el equipo de la figura 1 con velocidades de extracción de $30 \mathrm{~cm} / \mathrm{min}$.

La figura 5 muestra el incremento de masa por unidad de área en función de la densidad de corriente aplicada. La línea discontinua corresponde al incremento de masa obtenido por inmersión. Se aprecia que no hay variaciones sensibles en la masa de los depósitos obtenidos por inmersión y por deposición electroforética, lo cual sugiere que toda la masa depositada es consecuencia de la inmersión y que no hay aportación por EPD. Por tanto, el sistema no se comporta como una suspensión de partículas. Esto está de acuerdo con otros resultados obtenidos a partir de diferentes técnicas experimentales (RMN, SAXS, SANS...) [19]. Un sol catalizado en medio básico presenta una estructura de partículas discretas, que se comportan como partículas sólidas, mientras que un sol catalizado en medio ácido, como los utilizados en este trabajo, puede asimilarse a una estructura de "ovillos" de cadenas de sílice policondensadas cuyo índice de fractalidad es menor de 2, por lo que no se comporta como un sistema de partículas densas.

Se ha confirmado, por tanto, que para producir deposición electroforética a partir de soluciones sol-gel es necesaria la presencia de partículas densas que formen una suspensión estable.

A continuación se realizaron ensayos de deposición electroforética usando las suspensiones, preparadas a partir del sol híbrido catalizado con $\mathrm{HNO}_{3}$ a $\mathrm{pH} 3$ con distintas concentraciones de $\mathrm{SiO}_{2}$ coloidal aplicando densidades de corriente entre 0,16 a $0,8 \mathrm{~mA} / \mathrm{cm}^{2}$ durante una hora. La figura 6 muestra el incremento de masa por unidad de área en función de la densidad de corriente aplicada para cada una de las suspensiones. Las líneas punteadas corresponden al incremento de masa obtenido por inmersión para cada concentración.

Los resultados obtenidos para el sol híbrido que tiene un $1 \%$ de partículas son similares a los de inmersión, lo que implica que no hay deposición electroforética medible. Sólo con soles híbridos con contenidos de partículas de al menos

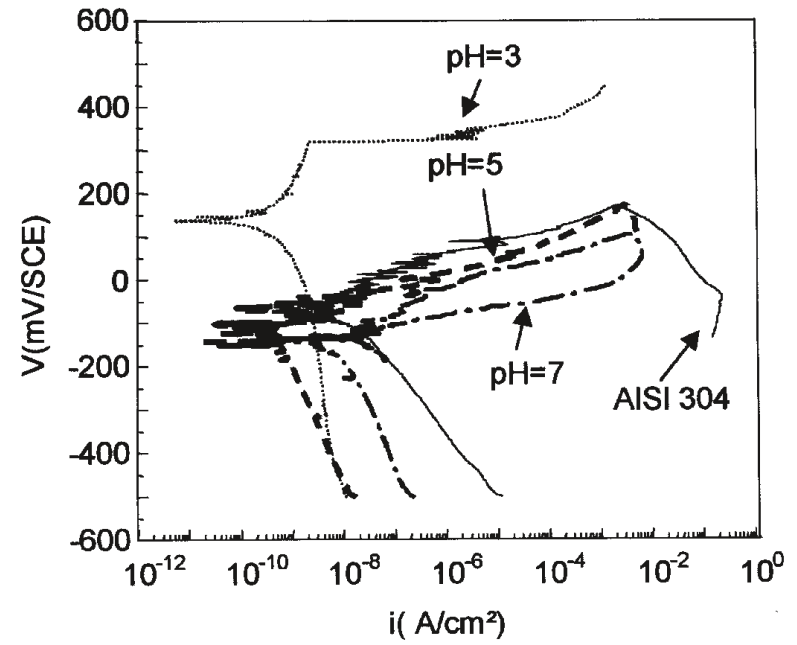

Figura 4. Curvas de polarización en $\mathrm{NaCl}$ 0,6 M de sustratos de acero inoxidable AISI 304 recubiertos por inmersión en soles híbridos preparados a diferentes $\mathrm{pH}$

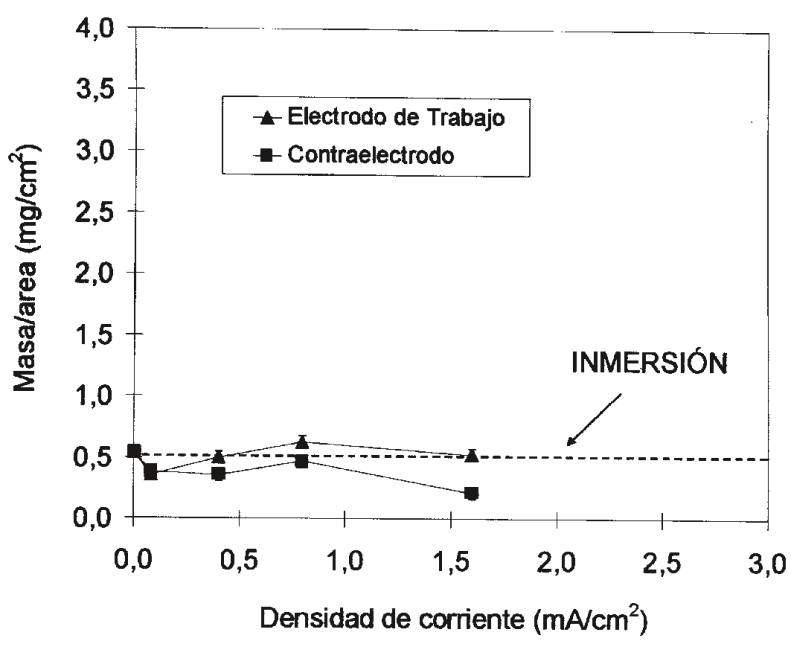

Figura 5. Variación de masa por unidad de área en función de la densidad de corriente en ensayos de EPD a partir de soles híbridos de $\mathrm{pH}=3$

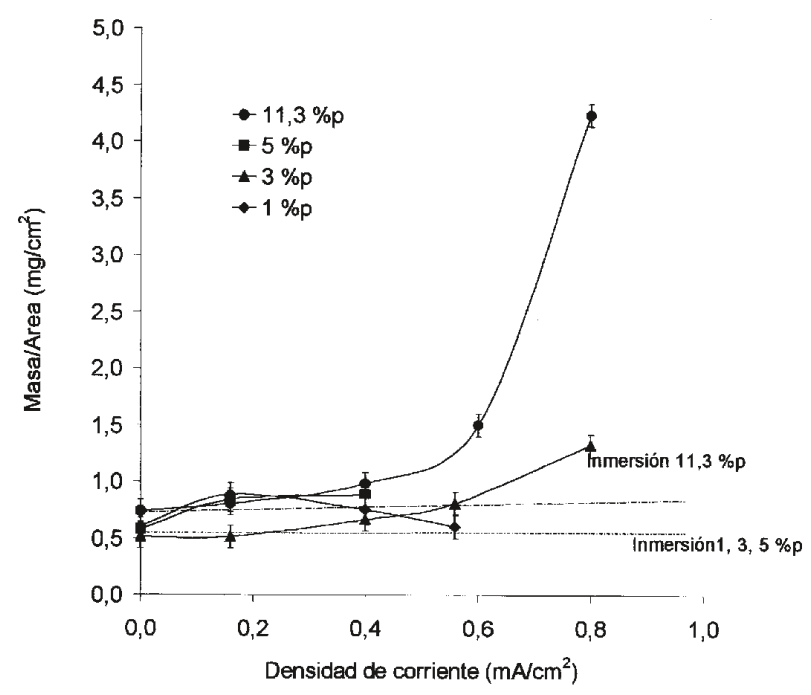

Figura 6. Variación de masa por unidad área en función de la densidad de corriente aplicada para suspensiones con 1, 3, 5 y $11 \%$ de partículas. 
$3 \%$ se puede observar un incremento de masa apreciable respecto al de inmersión, mostrando que el espesor de EPD es mayor que cero.

Aplicando una densidad de corriente de $0,8 \mathrm{~mA} \mathrm{~cm}^{-2}$ sobre los soles híbridos con un $3 \%$ de partículas se han conseguido recubrimientos homogéneos de hasta $6 \mu \mathrm{m}$ de espesor total $\left(\mathrm{e}_{\text {inmersión }}=1,5 \mu \mathrm{m}\right.$ y $\left.\mathrm{e}_{\mathrm{EPD}}=4,5 \mu \mathrm{m}\right)$, que presentan una buena adherencia al sustrato. A partir de la suspensión con un contenido en partículas de $11,3 \%$ se alcanzó un espesor máximo de $20 \mu \mathrm{m}$ pero el recubrimiento se agrietó durante el secado, despegándose del sustrato.

La figura 7 presenta un recubrimiento sobre acero inoxidable AISI 304 obtenido por EPD con una suspensión del 3 $\%$ de partículas después del tratamiento térmico de $450^{\circ} \mathrm{C} / 15$ minutos en flujo de $\mathrm{N}_{2}$. La observación del recubrimiento por microscopía óptica revela que las grietas nacen a partir de una aglomerado de partículas, siendo estos aglomerados el origen de defectos macroscópicos irreversibles.

Por otro lado, la aplicación de un campo eléctrico puede favorecer la corrosión de los sustratos metálicos, con el consiguiente deterioro de la superficie y por tanto, de las propiedades de los recubrimientos. Para evitar la corrosión se deberían aplicar potenciales inferiores al potencial de corrosión o, al menos, en la zona correspondiente al estado de pasivación. Por ello, se han determinado las curvas de polarización sobre el sol con el fin de evaluar el intervalo de densidades de corriente óptimo para llevar a cabo los ensayos de EPD sin dañar al metal. Aunque esto no siempre es factible porque la disminución del campo eléctrico reduce notablemente la cinética de EPD

El valor de densidad de corriente límite se determinó a partir de ensayos de voltametría utilizando el sol híbrido catalizado con ácido nítrico $(\mathrm{pH}=3)$. A partir de la curva de polarización obtenida, figura 8 , se puede determinar el valor de densidad de corriente de pasivación y por tanto la densidad de corriente límite. La ipasivación es aproximadamente $0,01 \mathrm{~mA} . \mathrm{cm}^{-2}$, por lo que a valores de intensidad mayores los sustratos se oxidan, mientras que si la intensidad es menor que $0,01 \mathrm{~mA} \mathrm{~cm}^{-2}$ se evita la corrosión durante el proceso de deposición electroforética.

Los resultados anteriores ponen de manifiesto la necesidad de incidir en dos líneas de actuación para aumentar el espesor de los recubrimientos y mejorar sus propiedades. Por un lado, la obtención de recubrimientos homogéneos de mayor espesor exige el estudio de las condiciones de preparación de suspensiones homogéneas de nanopartículas en soles híbridos y el control de la cinética de EPD. Por otra parte, el aspecto más novedosos de este trabajo se centra en la posibilidad de emplear la técnica de EPD, para conseguir obtener recubrimientos con espesores significativamente mayores respecto a los obtenidos por inmersión.

El objetivo a corto plazo es controlar la cinética de EPD para producir recubrimientos más gruesos aplicando potenciales inferiores al de corrosión, con el fin de eliminar los problemas derivados de la degradación del metal.

Aunque estos son los resultados preliminares, se ha demostrado que la técnica de EPD es una herramienta válida para obtener recubrimientos de mayor espesores que los obtenidos por la técnica de inmersión, lo que abre nuevos horizontes al procesamiento vía sol-gel. Los soles ácidos no se comportan como sistemas particulados, por lo que no se pueden depositar por EPD, pero la adicción de partículas al sol permite aumentar de forma significativa el espesor del recubrimiento por electrodeposición.

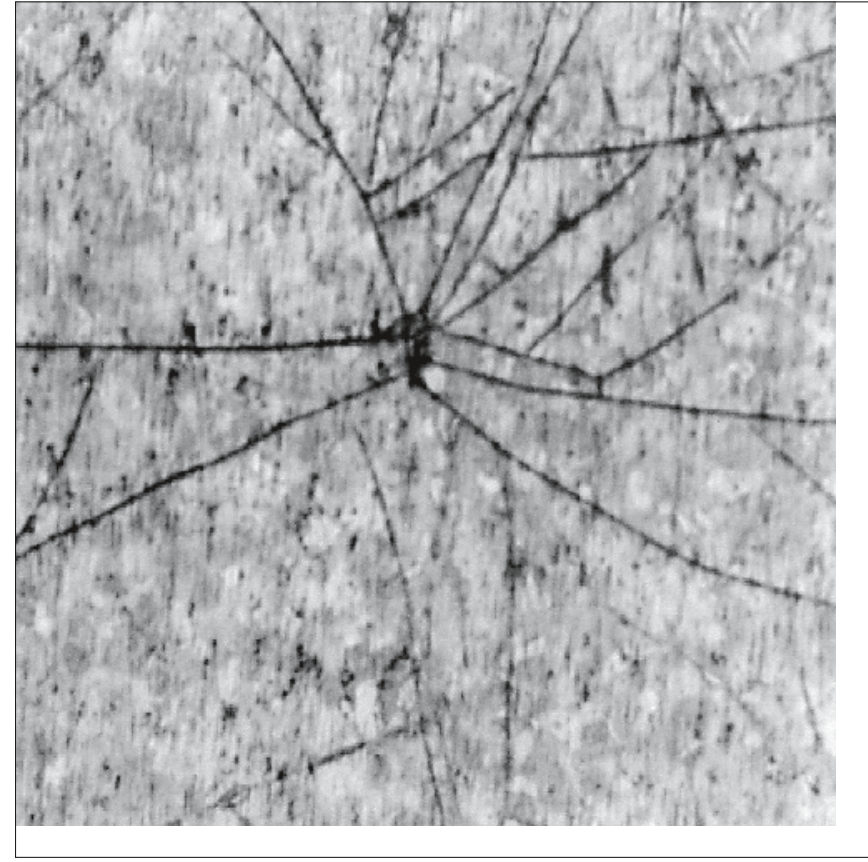

Figura 7. Recubrimiento vítreo sobre acero inoxidable AISI 304 obtenido a partir de una suspensión al $3 \%$ de partículas y tratado a $450^{\circ} \mathrm{C} / 15 \mathrm{~min}$ en aire.

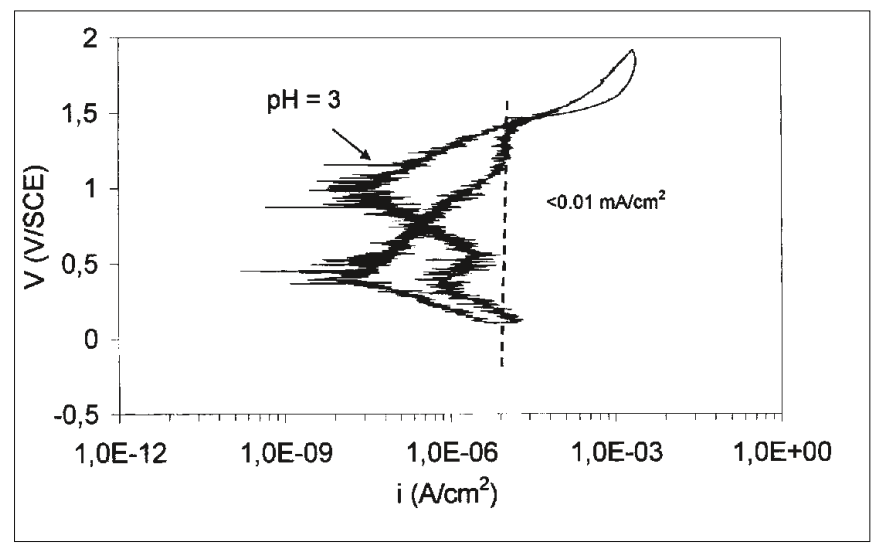

Figura 8. Curvas de polarización de soles híbridos de MTES $=40 / 60$ catalizados con $\mathrm{HNO}_{3}$ a $\mathrm{pH} 3$.

TEOS/

\section{CONCLUSIONES}

Se ha diseñado y puesto a punto un equipo para preparar recubrimientos por EPD a partir de soluciones sol-gel, que permite separar las dos contribuciones al espesor: la proveniente del proceso de inmersión y la producida por deposición electroforética.

Los soles catalizados en medio ácido no se depositan por EPD, indicando que es necesaria la presencia de partículas densas para producir deposición electroforética.

La medida de las curvas de polarización permite determinar el potencial de pasivación y, por tanto, la máxima densidad de corriente a aplicar durante la EPD para evitar la corrosión del sustrato metálico.

Es posible conseguir recubrimientos homogéneos y fuertemente adheridos al metal, con espesores aproximados de 6 $\mu \mathrm{m}$ a partir de soles híbridos con adición de nanopartículas de sílice. 


\section{AGRADECIMIENTOS}

Los autores agradecen la financiación parcial de este trabajo a través de los proyectos BRITE BE-97-5111, CICMT (MAT99-0872) y CAM (07N/0041/1999).

\section{BIBLIOGRAFÍA}

1. G. Wranglén. "An Introduction to Corrosion and Protection of Metals", Institut for Metallskydd, Estocolmo (Suecia), 1972

2. K. Isumi, M. Murakami, T. Deguchi, A. Morita, N. Tohge and T. Minami. "Zirconia Coating on Stainless Steel Sheets from Organozirconium Compounds". J. Amer. Ceramic. Soc. 72 14651468 (1989)

3. O. de Sanctis, N. Pellegri, A. Marajofsky, C. Parodi and A. Durán. “ Protective Glass Coatings on Metallic Substrates" J. Non-Cryst. Solids. 121 338-343 (1990).

4. S. Sakka, K. Kamiya, K. Makita and Y. Yamamoto. "Formation of Sheets and Coating Films from Alkoxide Solution" J. Non-Cryst Solids. 63 223-235 (1984).

5. J. J. Damborenea, N. Pellegri, O. de Sanctis, A. Durán. “Electrochemical Behaviour of $\mathrm{SiO}_{2}$ SolGel Coatings on Stainless Steel". J. Sol-Gel Sci. Tech. 239-244 (1995).

6. M. A. Villegas, M. Aparicio, A. Durán. "Thick Sol-Gel Coatings Based on the $\mathrm{B}_{2} \mathrm{O}_{3}-\mathrm{SiO}_{2}$ System". J. Non-Cryst. Solids. 218 146-150 (1997).

7. M. Mening, C. Schelle, A. Durán, J. J. Damborenea, M. Guglielmi and G. Brusatin. “Investigation of Glass-like Sol-Gel Coatings for Corrosion Protection of Stainless Steel against Liquid and Gaseous Attack". J. Sol-Gel Sc. Tech. 13 717-722 (1998).
8. M. Menning, G. Jonschker, H. Schimidt. "Sol-Gel Derived Thick Coatings and their Thermomechanical and C

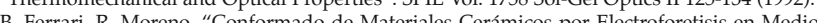
Acuosos". Bol. Soc. Esp. Ceram. Vidrio. 38 [5] 369-381 (1998).

10. R. Moreno, B. Ferrari. "Advanced Ceramic via EPD of Aqueous Slurries". Am. Ceram. Soc. Bull. 79 44-48 (2000)

11. W. J. Dalzell, D. E. Clark. "Thermophoretic and Electrophoretic Deposition of Sol-Gel Composite Coatings". Ceram. Eng Sci. Proc. 7 1014-1026 (1986).

2. P. Sarkar, P. S. Nicholson. "Electrophoretic Deposition (EPD): Mechanisms, Kinetics and Application to Ceramics". J. Am. Ceram. Soc. 78 [8] 1987-2002 (1996).

13. B. Ferrari, J. C. Fariñas, R. Moreno. "Determination and Control of Metallic Impurities in Alumina Deposits Obtained by Aqueous Electrophoretic Deposition". J. Am. Ceram. Soc., en prensa.

14. D. E. Clark, W. J. Dalzell and D. C. Folz. "Electrophoretic Alumina Sol-Gel Coatings on Metallic Substrates". Ceram. Eng. Sci. Proc. 9 1111-1118 (1988).

15. B. Cabot, A. Foissy. "Reversal of the surface charge of a mineral powder: application to electrophoretic deposition of silica for anticorrosion coatings". J. Mater. Sci. 33 3945-3952 (1998).

16. M. Aparicio, M. A. Villegas y A. Durán. "Protección antioxidante de materiales compuestos $\mathrm{C} / \mathrm{SiC}$ mediante recubrimientos de sílice por sol-gel". Bol. Soc. Esp. Ceram. Vidreo. 36, n 2-3, C/SiC mediante

17. M. Guglielmi, S. Zenezini, "The thickness of sol-gel silica coatings obtained by dipping". J Non-Cryst. Solids. 121 303-309 (1990)

18. M.G. Fontana, N.D. Greene. "Mineral Acids", pp 223-262 en Corrosion Engineering. McGrawHill Book Company, New York (USA), 1978

19. G. Brinker, G. W. Scherer. "Sol-Gel Science, The Physics and Chemistry of Sol-Gel Processing". Academic Press Ltda., London (UK), 1990

Recibido: 28-02-00

Aceptado: 8-05-00

$-$

\section{FE DE ERRATAS}

\section{En el Vol. 39 n'1, pag. 69, del trabajo "Abrasión de superficies de azulejos cerámicos esmaltados», de L. Espósito y A. Tucci del Centro Cerámico Italiano, apareció publicada la misma figura (2) en tres ocasiones. Seguidamente reproducimos la página completa con las figuras correctas.}

pectivamente. Un descenso en la porosidad cerrada y un aumento en la resistencia a la fractura mejoran la resistencia al desgaste por fricción. En las dos estructuras, la dependencia no es lineal, como también se encuentra en el desgaste erosivo húmedo de las cerámicas aluminosas (8)

Además, se debe hacer constar que a pesar de la conocida dependencia en la dureza que muestran los materiales frágiles $(6,7)$, se descubrieron diferentes repuestas de desgaste, tabla IV, incluso a pesar de que la dureza de la superficie de las muestras testadas era bastante parecida. Aparentemente este resultado es sorprendente. Es cierto, mientras que los valores de dureza de los Vickers de la superficie propia de los azulejos cerámicos esmaltavalores de dureza de los Vickers de la superficie propia de los azulejos cerámicos esmalta-
dos es bastante semejante (11), las características físicomecánicas del material a granel en dos es bastante semejante (11), las características físicomecánicas del material a granel en
una sección cruzada de la capa de esmalte son bastante diferentes, dependiendo de la tecnología de esmaltado, de la composición de la pasta mezclada, de las características del esmalte, de la reacciones químicas y del ciclo de cocción. En cuanto a esto, desde un valor de dureza máximo cerca de la superficie, muy parecido al encontrado en la propia superficie, en la sección cruzada de un azulejo esmaltado la dureza de la capa de esmalte desciende continuamente, llegando a un valor mínimo en la capa intermedia de la pasta de la capa rentes cerca de la capa jos cerámicos de monococción como en los de bicocción. Por esta razón, después de retirar las primeras capas de la superficie compactas y finas, sin poros, la carga abrasiva PEI entra en contacto progresivamente con un material menos denso, caracterizado, al contrario de lo que ocurre con la superfiice, por dos distribuciones de defectos distintas: i) los defectos que ya existían debido a la porosidad atrapada en la capa de esmalte durante el proceso de cocción y, ii) el deterioro provocado, las rayas, cortes y eliminación de material, como consecuencia de la acción abrasiva. Mientras que la porosidad, generalmente debida a los poros esféricos, y el tamaño medio del poro se pueden evaluar fácilmente, el desgaste producido por el deterioro es difícil de detectar y en cualquier caso es diferente en la morfología y la geometrí. desgaste producido por el deterioro es una función de las características de los materiales y de los parámetros de ensayo, en este caso el número de revoluciones.

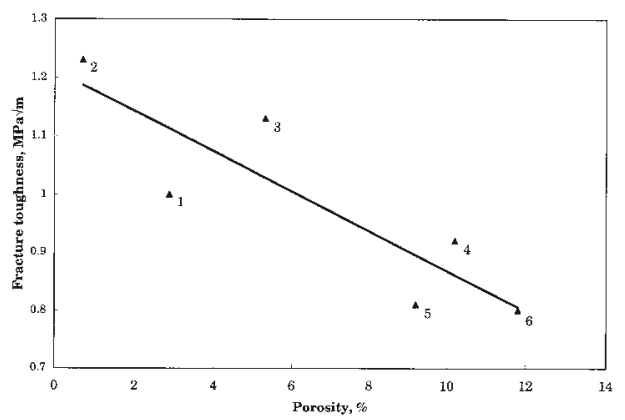

Figura 2 - Resistencia a la fractura como una función de la porosidad de las capas de esmalte: los números de la estructura corresponden a las muestras.

Figure 2 - Fracture toughness as a function of the porosity of the glaze layers: the numbers in the plot correspond to the samples. the cross section of a glazed tile the hardness of the glaze layer continually decreases, reaching a minimum value at the glaze layer-ceramic body interface (19). Even with different fracture mechanisms near the interface, this behaviour takes place in both single-fired and double-fired ceramic tile. Thus, after the first thin compact surface layers, without pores, have been removed, the abrasive PEI charge progressively comes into contact with less dense material, characterised, contrary to the surface by two differt flaw distions glaze layer during the firing process and, ii) the induced damage, scratches, cuts and material removal, as a consequence of the abrasive action. While the porosity, generally due to spherical pores, and $\mathrm{val}$, as a consequence of the abrasive action. While the porosity, generally due to spherical pores, and
the average pore size can be easily evaluated, the wear induced damage is difficult to detect and in any case different in morphology and geometry. Wear induced damage is a function of

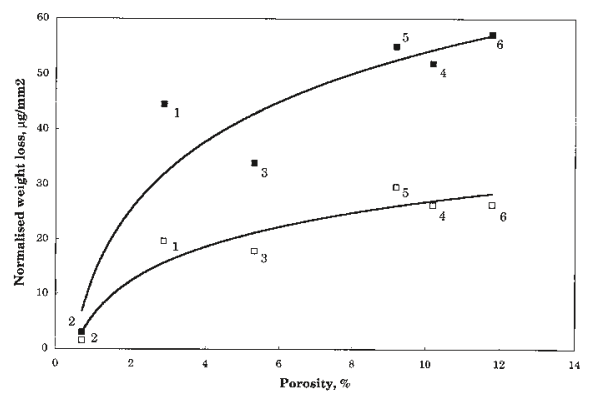

Figura 4 - NWL como una función de resistencia a la fractura: los números de la estructura corresponden a las muestras. $6000(\mathrm{O}), 12000 \mathrm{rev}$. (๑).

Figure 4 - NWL as a function of fracture toughness: the numbers in the plot correspond to the samples. 6000 rev. (O), $12000 \mathrm{rev}$. (•)

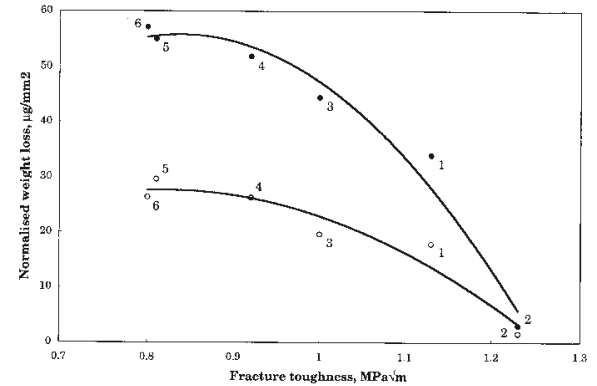

Figura3 - NWL como una función de la porosidad de las capas de esmalte: los números de la estructura corresponden a las muestras. 6000 rev. $\quad(\square), 12000$ revv. (口)

Figure 3 - NWL as a function of the porosity of the glaze layers: the numbers in the plot correspond to the samples. $6000 \mathrm{rev}$ ( $\square$ ), $12000 \mathrm{rev}$. ( 